\title{
Universal function of the non-equilibrium phase transition of nonlinear Pólya urn
}

\author{
Kazuaki Nakayama* \\ Department of Mathematics, Faculty of Science, Shinshu University, \\ Asahi 3-1-1, Matsumoto, Nagano 390-8621, Japan \\ Shintaro Morit \\ Department of Mathematics and Physics, \\ Faculty of Science and Technology, Hirosaki University, \\ Bunkyo-cho 3, Hirosaki, Aomori 036-8561, Japan
}

(Dated: November 17, 2020)

\begin{abstract}
We study the phase transition and the critical properties of a nonlinear Pólya urn, which is a simple binary stochastic process $X(t) \in\{0,1\}, t=1, \cdots$ with a feedback mechanism. Let $f$ be a continuous function from the unit interval to itself, and $z(t)$ be the proportion of the first $t$ variables $X(1), \cdots, X(t)$ that take the value $1 . X(t+1)$ takes the value 1 with probability $f(z(t))$. When the number of stable fixed points of $f(z)$ changes, the system undergoes a non-equilibrium phase transition and the order parameter is the limit value of the autocorrelation function. When the system is $Z_{2}$ symmetric, that is, $f(z)=1-f(1-z)$, a continuous phase transition occurs, and the autocorrelation function behaves asymptotically as $\ln (t+1)^{-1 / 2} g(\ln (t+1) / \xi)$, with a suitable definition of the correlation length $\xi$ and the universal function $g(x)$. We derive $g(x)$ analytically using stochastic differential equations and the expansion about the strength of stochastic noise. $g(x)$ determines the asymptotic behavior of the autocorrelation function near the critical point and the universality class of the phase transition.

PACS numbers: $05.70 . \mathrm{Fh}, 89.65 . \mathrm{Gh}$
\end{abstract}

\footnotetext{
*nakayama@math.shinshu-u.ac.jp

$\dagger$ shintaro.mori@hirosaki-u.ac.jp
} 


\section{INTRODUCTION}

Before the birth of econophysics [1], Brian Arthur studied increasing returns or positive feedback in economies and demonstrated that they can magnify small perturbations in the market [2, 3]. Let us assume that two selectively neutral technologies simultaneously enter the market. Owing to network externality, the utility of a product becomes an increasing function of its market share. An initial small imbalance in the market share can eventually induce catastrophic imbalance. A similar mechanism also applies when people make decisions in an uncertain environment [4]. In this case, it is rational to adopt the majority choice among previously chosen options, as this reflects the wisdom of the crowd [5]. The tendency to adopt the majority choice, overriding one's own private signal, is called information cascade or rational herding [4]. Positive feedback from previous choices affects later choices, and an initial small imbalance in the choices can have similar effects to those of increasing returns in the market.

To describe the final catastrophic imbalance caused by the increasing returns and the information cascade, a non-linear Pólya urn model was adopted [6]. In the original Pólya urn model, an urn consists of $t$ balls, where the proportion of red balls is $z(t) \in(0,1)$, and the rest of the balls are blue [7]. The probability of a new red ball being added to the urn is $z(t)$, whereas the corresponding probability for a blue ball is $1-z(t)$; the proportion of red balls then becomes $z(t+1)$. This procedure is iterative, and $z(t)$ follows the beta distribution in the limit as $t \rightarrow \infty$. In nonlinear generalizations of this model, a continuous function $f:[0,1] \rightarrow[0,1]$ determines the probability $f(z(t))$ of a red ball being added at stage $t+1$. This nonlinear version is referred to as a nonlinear Pólya process [6, 8]. In contrast to the original linear model, the nonlinear model can have isolated stable states. The fixed point $z_{*}$ of $f(z)$, where $f\left(z_{*}\right)=z_{*}$, is (un)stable if $f^{\prime}\left(z_{*}\right)$ is smaller (greater) than 1 [6]. $z_{*}$ is referred to as downcrossing (upcrossing), as the graph $y=f(z)$ crosses the curve $y=z$ in the downward (upward) direction if $f^{\prime}\left(z_{*}\right)$ is smaller (larger) than 1. When $f(z)$ touches the diagonal in the $(z, q)$ plane at $z_{t}, z_{t}$ is referred to as the touchpoint. The stability of $z_{t}$ depends on the difference between the slope of $f(z)$ and the diagonal $z[8]$.

The market share $z$ of a product determines the probability $f(z)$ that a new customer adopts it. An S-shaped $f(z)$ function with two stable fixed points suggests the random monopoly formed when a technology dominates over the other depends on initial chance 
fluctuations. This is a type of butterfly effect or high sensitivity to initial conditions in chaos theory; however, the final states are restricted to stable fixed states. An information cascade experiment provides a concrete setup for the physical realization of the formation of the final imbalance [9]. The change in the number of stable states can be observed by controlling the uncertainty of the subjects [10, 11].

In statistical physics, two aspects of non-linear Pólya urns have been studied. The supernormal transition in the convergence of $z(t)$ to a unique stable fixed point $z_{*}$ was studied in the context of long-range correlations in time series of financial data and DNA sequences [12]. Another problem is to understand the change in the number of stable states as a non-equilibrium phase transition [13, 14]. The order parameter is the limit value of the autocorrelation function. If this value is zero, the memory of the past disappears in future variables. If it is positive, the memory of past variables remains and affects future variables forever.

The critical behavior of the autocorrelation function bears a strong resemblance to the order parameters of absorbing-state phase transitions [15]. If $f(z)$ is given as the superposition of a constant function and a step function as $(1-p) q+p \theta(z-1 / 2), q \neq 1 / 2,0<p<1$ (we term this the digital model), a continuous phase transition occurs at $p=p_{c}(q)=1-1 / 2 q[16]$. For $p<p_{c}(q)$, there is only one stable state at $z_{*}=(1-p) q+p$, and the correlation function decays exponentially. At the critical point $p=p_{c}(q)$, where there is a stable fixed point at $z_{*}=(1-p) q+p$ and an unstable touchpoint at $z_{t}=(1-p) q$, the autocorrelation function exhibits power-law decay. For $p>p_{c}(q)$, there are two stable states $z_{ \pm}$at $(1-p) q+p,(1-p) q$, and the order parameter becomes positive [13]. Furthermore, the asymptotic behavior of the autocorrelation function obeys a scaling law, namely, $b(q) t^{-1 / 2} g(t / \xi(q, p))$, with universal function $g(x)$ [14]. If $f(z)$ is a smooth function, the system undergoes a continuous phase transition when $f(z)=1-f(1-z)$. The critical and the off-critical behavior of the autocorrelation function are completely different from those of the aforementioned digital model. At the critical point, the autocorrelation function decays as $\sim \ln t^{-1 / 2}$. Below the critical point, the autocorrelation function exhibits power-law decay in $t$, and the power-law exponent is determined as $f^{\prime}\left(z_{*}\right)-1$. Based on the behaviors and the analogy with the digital model, we propose the scaling form $b(\ln t)^{-1 / 2} g(\ln t / \xi)$ for the autocorrelation function. Here, we define the correlation length $\xi$ using the exponent of the power-law decay as $\xi=1 /\left(1-f^{\prime}\left(z_{*}\right)\right)$. 
In this study, we derive the universal function $g(x)$ in the case where $f(z)$ is a smooth function as above. We map the stochastic process to a stochastic differential equation (SDE) and expand its trajectory about the strength of the noise. We solve the initial value problem and analytically derive the autocorrelation function. Then, we derive $g(x)$ and verify it numerically. The paper is organized as follows. We define the model in Section II, and map it to an SDE and expand its trajectory about the strength of the noise in Section III. In Section IV, we solve the corresponding initial value problem, estimate the asymptotic behavior of the autocorrelation function, and derive the universal function. In Section $\mathrm{V}$, we numerically estimate the autocorrelation and the universal function, and we verify that the derived universal function completely describes the numerical results. We summarize the results in Section VI.

\section{MODEL}

We define a binary stochastic process $X(t) \in\{0,1\}, t \in\{1,2, \cdots, T\}$, where the probability that $X(t)$ takes the value 1 is given by a function $f(z, h)$ of the proportion $z(t-1)$ of the variables $X(1), \cdots, X(t-1)$ that are equal to 1 .

$$
\begin{aligned}
f(z, h) & \equiv \mathrm{P}(X(t)=1 \mid z(t-1)=z)=z-(z-1 / 2)^{3}+p(z-1 / 2)+h \\
z(t) & =\frac{1}{t} \sum_{s=1}^{t} X(s) \text { for } t>0, \text { and } z(0)=\frac{1}{2} .
\end{aligned}
$$

The choice of $f(z, h)$ is arbitrary, and we adopt the above form. In the numerical studies, we adopt another form for $f(z, h)$ to verify that the results are independent of the choice of $f(z, h)$. With this choice for $f(z, h)$, we can estimate the fixed point of $f(z, h)$ by solving $f(z)=z$ explicitly. Here, we consider the parameter space $(p, h)$, where $0 \leq f(z, h) \leq 1$ for $\forall 0 \leq z \leq 1$.

The number of stable fixed points depends on $(p, h)$, and there is a threshold value $p=p_{c}(h)$, which is a function of $h$ (Fig. 1):

$$
p_{c}(h)=3(h / 2)^{2 / 3}
$$

For $p<p_{c}(h)$, there is only one fixed point at $z=z_{*}$. As $p$ increases and $h$ is fixed, $f(z, h)$ 


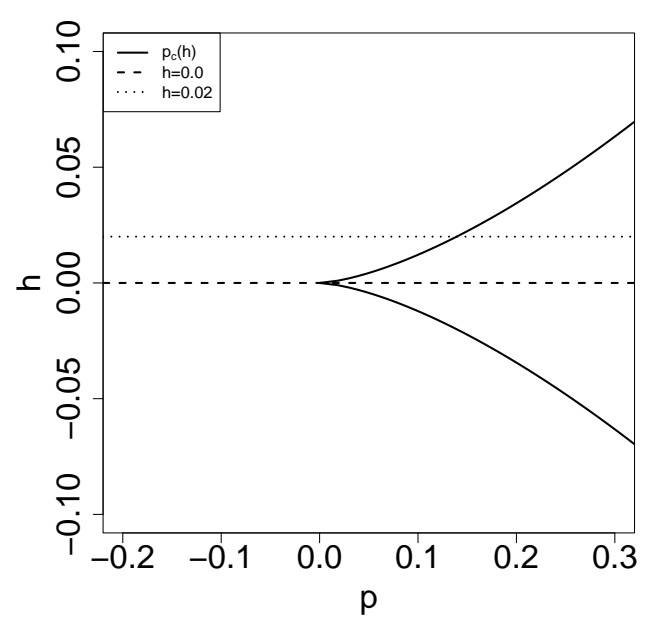

FIG. 1. Phase diagram in $p-h$ plane. The phase boundary is given by (2). For $p<p_{c}(h)$, there is a stable fixed point at $z_{*}$. For $p>p_{c}(h)$, there are two stable fixed points at $z_{ \pm}$. For $h=0, p=p_{c}(0)=0$, there is a touchpoint at $z=0$. For $h \neq 0$ and $p=p_{c}(h)$, there is a stable fixed point at $z_{*}$, and a touchpoint at $z_{t}$.

becomes tangential to the diagonal at $z_{t}$ for $p=p_{c}(h)$. For $h \neq 0, z_{t} \neq z_{*}$, and both $z_{t}$ and $z_{*}$ are stable. For $h=0, z_{t}$ and $z_{*}$ are equal and stable. In both cases, the slope of the curve at $z_{t}$ is equal to 1 . For $p>p_{c}(h)$, there are three fixed points, and we denote them as $z_{-}<z_{u}<z_{+} ; z_{-}$and $z_{+}$are stable, whereas $z_{u}$ is unstable. We denote the slope of $f(z, h)$ at $z_{*}$ and $z_{ \pm}$by $l_{*}$ and $l_{ \pm}$, respectively. As $z_{*}$ and $z_{ \pm}$are stable and downcrossing, $l_{*}, l_{ \pm}<1$.

The autocorrelation function $C(t)$ (correlation function for brevity) is defined as the difference of the two conditional probabilities on $X(1)$ :

$$
C(t)=\mathrm{P}(X(t+1)=1 \mid X(1)=1)-\mathrm{P}(X(t+1)=1 \mid X(1)=0) .
$$

$C(t)$ can be defined as $\operatorname{Cov}(X(1), X(t+1)) / \mathrm{V}(X(1))$ using the variance of $X(1)$ and the covariance of $X(1)$ and $X(T+1)$. The asymptotic behavior of $C(t)$ depends on $(p, h)$. In the following, we analytically derive this behavior by mapping the model to an SDE. 


\section{MODEL ANALYSIS}

\section{A. Stochastic Differential Equation}

The random variable $z(t)$ defined in (1) satisfies the following recursion relation:

$$
z(t+1)=z(t)+\frac{X(t+1)-z(t)}{t+1}
$$

The conditional expectation and the conditional variance of $z(t)$ are estimated as follows:

$$
\begin{aligned}
& E(z(t+1) \mid z(t)=z)=z+\frac{f(z, h)-z}{t+1} \\
& V(z(t+1) \mid z(t)=z)=\frac{f(z, h)\{1-f(z, h)\}}{(t+1)^{2}} .
\end{aligned}
$$

The second term on the right-hand side of (5a) is regarded as a drift term, whereas the right-hand side of (5b) is interpreted as a diffusion coefficient. Thus, we introduce the continuous-time model described by the following Ito-type SDE:

$$
d z=\frac{f(z, h)-z}{t+1} d t+\frac{\sqrt{f(z, h)\{1-f(z, h)\}}}{t+1} d W_{t}
$$

where $W_{t}$ is the Wiener process [17]. In the following, we study the following simplified SDE model:

$$
d z=\frac{f(z, h)-z}{t+1} d t+\frac{D}{t+1} d W_{t}
$$

where $D$ is a small positive constant. It seems that there is no significant difference between (6) and (17) as far as the long-time behavior of the system is concerned[18].

\section{B. Small Noise Approximation}

Let $y=z-1 / 2$ be a new random variable. We define $g(y, h)=[f(z, h)-z]_{z=y+1 / 2}=$ $-y^{3}+p y+h$. Then, Eq. (17) becomes

$$
d y=\frac{g(y, h)}{t+1} d t+\frac{D}{t+1} d W_{t}
$$


We expand $y(t)$ in powers of $D$ :

$$
y(t)=y_{0}(t)+D y_{1}(t)+D^{2} y_{2}(t)+\cdots
$$

Then, Eq. (8) is solved recursively. As we are interested in the correlation function $C(t)$, we adopt the initial condition $y(0)= \pm 1 / 2$, that is,

$$
y_{n}(0)= \pm \frac{1}{2} \delta_{n 0} \quad(n=0,1,2, \ldots)
$$

For simplicity, $y^{ \pm}(t)$ and $y_{n}^{ \pm}(t)$ denote the solutions corresponding to each initial condition.

We summarize the solution of the initial value problem corresponding to (8). The details of the calculation are given in Appendix A. We first note that the classical solution $y_{0}(t)$ of (8) is independent of $D$. It is easily obtained as an implicit function:

$$
\ln (t+1)=\int_{ \pm 1 / 2}^{y_{0}^{ \pm}} \frac{d y}{g(y, h)}
$$

Furthermore, the expectation value and the variance of $y(t)$ are expressed, up to the second order of $D$, as

$$
\begin{aligned}
& E\left(y^{ \pm}(t)\right)=y_{0}^{ \pm}(t)+D^{2} E\left(y_{2}^{ \pm}(t)\right), \\
& V\left(y^{ \pm}(t)\right)=D^{2} E\left(y_{1}^{ \pm}(t)^{2}\right) .
\end{aligned}
$$

Finally, these expectation values are explicitly obtained as follows:

$$
\begin{aligned}
& E\left(y_{1}^{ \pm}(t)^{2}\right)=e^{2 G^{ \pm}(t)} \int_{0}^{t} \frac{e^{-2 G^{ \pm}(t)}}{(t+1)^{2}} d t \\
& E\left(y_{2}^{ \pm}(t)\right)=-3 e^{G^{ \pm}(t)} \int_{0}^{t} \frac{e^{-G^{ \pm}(t)} y_{0}^{ \pm}(t) E\left(y_{1}^{ \pm}(t)^{2}\right)}{t+1} d t
\end{aligned}
$$

where

$$
G^{ \pm}(t)=p \ln (t+1)-3 \int_{0}^{t} \frac{x_{0}^{ \pm}(t)^{2}}{t+1} d t
$$




\section{CORRELATION FUNCTION AND UNIVERSAL FUNCTION}

\section{A. Correlation Function and Order Parameter}

The correlation function $C(t)$ (defined in (3) ) is expressed as [14]

$$
C(t)=E\left(y^{+}(t)+g\left(y^{+}(t), h\right)\right)-E\left(y^{-}(t)+g\left(y^{-}(t), h\right)\right) .
$$

Let $a_{-} \leq a_{+}$be the stable fixed points of $g(y, h)$. The order parameter $c=c(p, h)$ is the difference $a_{+}-a_{-}$of the stable fixed points because $y^{ \pm}(t) \rightarrow a_{ \pm}$as $t \rightarrow \infty$ up to $O\left(D^{2}\right)$.

Our result is that

$$
c(p, h)= \begin{cases}2 \sqrt{p} \cos \frac{\pi-2 \mu(p, h)}{6}, & p \geq p_{c}(h)=3(h / 2)^{2 / 3}, \\ 0, & p<p_{c}(h)=3(h / 2)^{2 / 3}\end{cases}
$$

where

$$
\mu(p, h)= \begin{cases}\arccos \left\{\frac{|h|}{2}\left(\frac{3}{p}\right)^{3 / 2}\right\}, & h \neq 0 \\ \frac{\pi}{2}, & h=0 .\end{cases}
$$

Figure 1 shows the phase diagram in the $p-h$ plane. The ordered phase with $c(p, h)>0$ is the region where $p \geq p_{c}(h)=2(p / 3)^{3 / 2}$.

The correlation function $C(t)$ is

$$
\begin{aligned}
& C(t)=C_{0}(t)+D^{2} C_{2}(t) \\
& C_{0}(t)= y_{0}^{+}(t)-y_{0}^{-}(t)+g\left(y_{0}^{+}(t), h\right)-g\left(y_{0}^{-}(t), h\right), \\
& C_{2}(t)=E\left(y_{2}^{+}(t)\right)-E\left(y_{2}^{-}(t)\right)+\left\{g_{y}\left(y_{0}^{+}(t), h\right) E\left(y_{2}^{+}(t)\right)-g_{y}\left(y_{0}^{-}(t), h\right) E\left(y_{2}^{-}(t)\right)\right\} \\
& \quad+\frac{1}{2}\left\{g_{y y}\left(y_{0}^{+}(t), h\right) E\left(y_{1}^{+}(t)^{2}\right)-g_{y y}\left(y_{0}^{-}(t), h\right) E\left(y_{1}^{-}(t)^{2}\right)\right\},
\end{aligned}
$$

where $C_{0}(t)$ and $C_{2}(t)$ represent the $O\left(D^{0}\right)$ and $O\left(D^{2}\right)$ parts of the function $C(t)$, respectively. 


\section{B. Correlation Function in the Non-symmetric Domain}

We summarize the asymptotic behavior of the correlation function when $h>0$.

a. Ordered Phase In the off-critical region $p>3(h / 2)^{2 / 3}$, the correlation function exhibits power-law decay:

$$
C(t) \sim c(p, h)+c^{\prime}(t+1)^{-\zeta},
$$

where $c^{\prime}$ is a constant, and

$$
\zeta=p+2 p \sin \frac{4 \mu(p, h)-\pi}{6} .
$$

The function $\mu(p, h)$ is defined in (16).

On the critical line $p=3(h / 2)^{2 / 3}$, the function exhibits logarithmic behavior:

$$
C(t) \sim \sqrt{3 p}+\frac{1}{\sqrt{3 p} \ln (t+1)+c^{\prime}} .
$$

b. Disordered Phase When $p<3(h / 2)^{2 / 3}$, the correlation function behaves as follows:

$$
C(t) \sim c^{\prime} \cdot \begin{cases}(t+1)^{-\zeta}, & \zeta \neq 1 \\ (t+1)^{-2} \ln (t+1), & \zeta=1\end{cases}
$$

where

$$
\zeta= \begin{cases}p\left(3 \cosh ^{2} \frac{\mu}{3}+\sinh ^{2} \frac{\mu}{3}\right), & p>0, \\ |p|\left(3 \sinh ^{2} \frac{\mu}{3}+\cosh ^{2} \frac{\mu}{3}\right), & p<0, \\ 3 h^{2 / 3}, & p=0,\end{cases}
$$

and the parameter $\mu=\mu(p, h)$ is given by

$$
\mu(p, h)= \begin{cases}\cosh ^{-1}\left\{\frac{h}{2}\left(\frac{3}{p}\right)^{3 / 2}\right\}, & p>0 \\ \sinh ^{-1}\left\{\frac{h}{2}\left(\frac{3}{|p|}\right)^{3 / 2}\right\}, & p<0 .\end{cases}
$$




\section{Correlation Function in the Symmetric Domain}

When $h=0$, exact solutions can be obtained. The off-critical correlation function is estimated as

$$
C(t ; p)=2 \sqrt{\frac{p}{1-(1-4 p)(t+1)^{-2 p}}}+O\left(D^{2}\right), \quad p \neq 0
$$

whereas the on-critical correlation function is given by

$$
C(t ; 0)=\frac{1}{\sqrt{(1 / 2) \ln (t+1)+1\}}}+O\left(D^{2}\right) .
$$

The function $C(t ; p)$ is continuous, that is, $\lim _{p \rightarrow \pm 0} C(t ; p)$ coincides with $C(t ; 0)$.

\section{Universal Function}

In the ordered phase, the asymptotic behavior of the correlation function (24a) is $C(t)-$ $c(p, 0) \sim(t+1)^{-2 p}=e^{-2 p \ln (t+1)}$. The correlation length $\xi$ on the $\ln (t+1)$ scale is $1 / 2 p$ and diverges at $p=0$. Therefore, keeping $x=2 p \ln (t+1)$ constant and taking the limit as $\ln (t+1), \xi=1 / 2 p \rightarrow \infty$, we have the universal function

$$
g(x)=\lim _{p \rightarrow+0} \frac{C(t ; p)}{C(t ; 0)}=\sqrt{\frac{x}{1-e^{-x}}} .
$$

By contrast, in the disordered phase, the asymptotic behavior of the correlation function (24b) is $C(t) \sim(t+1)^{-|p|}=e^{-|p| \ln (t+1)}$. Therefore, $x=|p| \ln (t+1)$ should be constant to estimate the universal function. Thus,

$$
g(x)=\lim _{p \rightarrow-0} \frac{C(t ; p)}{C(t ; 0)}=\sqrt{\frac{2 x}{e^{2 x}-1}} .
$$

In the ordered phase $(p>0), g(x) \propto x^{1 / 2}$ for large $x$. As $C(t)=C(t ; 0) g(2 p \ln (t+1))$ and $C(t ; 0) \propto \ln (t+1)^{-1 / 2}$ for $\ln (t+1)>>1$, we have $C(t) \propto p^{1 / 2}$. The critical exponent $\beta$ for the order parameter $c \propto p^{\beta}$ is $1 / 2$, which is suggested based on the scaling hypothesis for $C(t)[14]$.

We also consider the universal function in the $h$ direction. Let $C(t ; p, h)$ be the correlation 
function. By (21), we have $C(t ; 0, h) \sim c^{\prime}(t+1)^{-3 h^{2 / 3}}$ when $h>0$ is small. Thus, we take the limit as $\ln (t+1), \xi=1 / 3 h^{2 / 3} \rightarrow \infty$ and keep the quantity $x=3 h^{2 / 3} \ln (t+1)$ constant. Then,

$$
g_{h}(x)=\lim _{h \rightarrow+0} \frac{C(t ; 0, h)}{C(t ; 0,0)}=\frac{\sqrt{x}}{2 \sqrt{2}}\left\{\tan \varphi^{-1}\left(e^{-x} \varphi(\pi / 2)\right)-\tan \varphi^{-1}\left(e^{-x} \varphi(-\pi / 2)\right)\right\}
$$

where the monotonically increasing function $\varphi(\theta)$ is defined as follows:

$$
\varphi(\theta)=e^{-\sqrt{3} \theta} \sin \left(\theta-\frac{\pi}{3}\right), \quad|\theta| \leq \frac{\pi}{2}
$$

\section{NUMERICAL STUDY OF PHASE TRANSITION}

We perform numerical integration of the master equation and estimate $C(t)$. We denote the probability function for $\sum_{s=1}^{t} X(s)$ with initial condition $X(1)=x_{1}$ as $P\left(t, n \mid x_{1}\right) \equiv$ $\mathrm{P}\left(\sum_{s=1}^{t} X(s)=n \mid X(1)=x_{1}\right)$. We have that $P\left(1, n \mid x_{1}\right)=\delta_{n, x_{1}}$ holds. The master equation for $P\left(t, n \mid x_{1}\right)$ is

$$
P\left(t+1, n \mid x_{1}\right)=f((n-1) / t, h) \cdot P\left(t, n-1 \mid x_{1}\right)+(1-f(n / t, h)) \cdot P\left(t, n \mid x_{1}\right) .
$$

We impose the boundary conditions $P\left(t, n, x_{1}\right)=0$ for $n<0$ or $n>t$. Using $P\left(t, n \mid x_{1}\right)$, we estimate $C(t)$ for $t \leq T=10^{6}$ as

$$
C(t \mid p, h)=\sum_{n=0}^{t} f(n / t, h)(P(t, n \mid 1)-P(t, n \mid 0))
$$

We set $h \in\{0.0,0.02\}$ and $p \in[-0.2,0.2]$. The plot of $C(T \mid p, h)$ vs. $p$ as well as the plot of $c(p, h)$ from Eq. (15) are shown in Fig. 2.

It is seen that $C(T \mid p, h)$ for $T=10^{6}$ is rather different from $c(p, h)=\lim _{T \rightarrow \infty} C(T \mid p, h)$ near $p=p_{c}(h)$. One cannot observe the transition between the phase with $c=0$ for $p<p_{c}(h)$ and that with $c>0$ for $p>p_{c}(h)$. This discrepancy can be accounted for by the limited system size $T$ and the strong correlation near $p=p_{c}(h)$. To see the thermodynamic $\operatorname{limit} T \rightarrow \infty$ and the phase transition, it is necessary to study the scaling function $g(x)$.

We numerically estimate $g(x)$ defined in Eq. (25). We estimate $C(T \mid p, 0) / C(t \mid 0,0)$ 


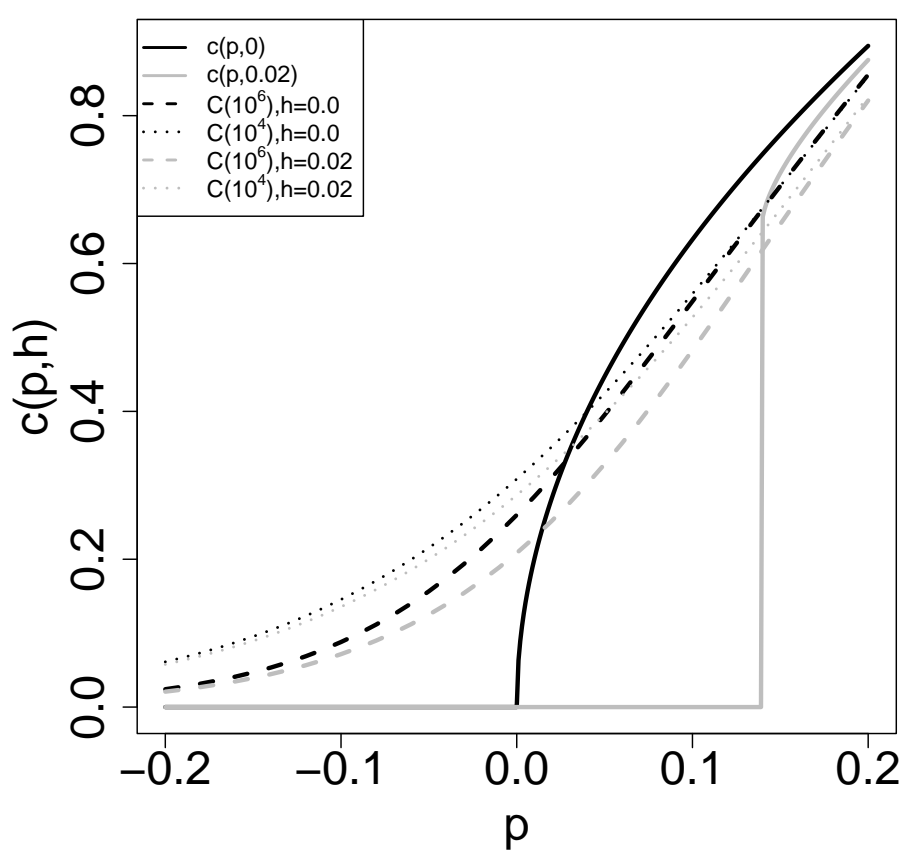

FIG. 2. Plot of $C\left(T=10^{6} \mid p, h\right)$ vs. $p$. We set $h=0.0(\square), 0.02(\circ)$ and $p \in[-0.2,0.2]$. The result of Eq. (15) is also plotted with solid and dotted curves.

for $\xi(p)>>1$ as a function of $x=T / \xi(p)$. We set $p \in[-0.01,0.01]$, [-0.001,0.001], $[-0.0001,0.0001]$. We also estimate $x(p) \equiv T / \xi(p)$ using Eq. (28).

$$
\xi(p)=\left\{\begin{array}{l}
1 /|p| p<0 \\
1 / 2 p \quad p>0
\end{array}\right.
$$

In addition to the model in Eq. (11), we also study another model with the following function:

$$
\left.f(z)=\frac{1}{2}\{\tanh (2 J(z-1 / 2)+h)+1)\right\}
$$

A continuous phase transition occurs at $h=0, J=1$. For $h=0$ and $J<1, z_{*}=1 / 2$ and $l_{*}=$ $f^{\prime}\left(z_{*}\right)=J$. For $h=0$ and $J>1$, there are stable states $z_{ \pm}$and $l_{ \pm}=J / \cosh ^{2}\left(2 J\left(z_{ \pm}-1 / 2\right)\right)$. The correlation length $\xi(J, 0)$ is defined as

$$
\xi(J)=\left\{\begin{array}{cc}
1 /(1-J) & J<1 \\
1 /\left(1-J / \cosh ^{2}\left(2 J\left(z_{ \pm}-1 / 2\right)\right)\right. & J>1
\end{array}\right.
$$


We set $J \in[0.99,1.01],[0.999,1.001],[0.9999,1.0001]$ and estimate $C(T \mid J, 0) / C(T \mid 1,0)$ for $T=10^{6}$. We also estimate $x(J) \equiv T / \xi(J)$ using Eq. (30) .

The plots of $C(T \mid p, 0) / C(T \mid 0,0)$ vs. $x(p)$ and $C(T \mid J, 0) / C(T \mid 1,0)$ vs. $x(J)$ are shown in Fig. 3, It is observed that the numerical results are well described by Eq. (25). It can
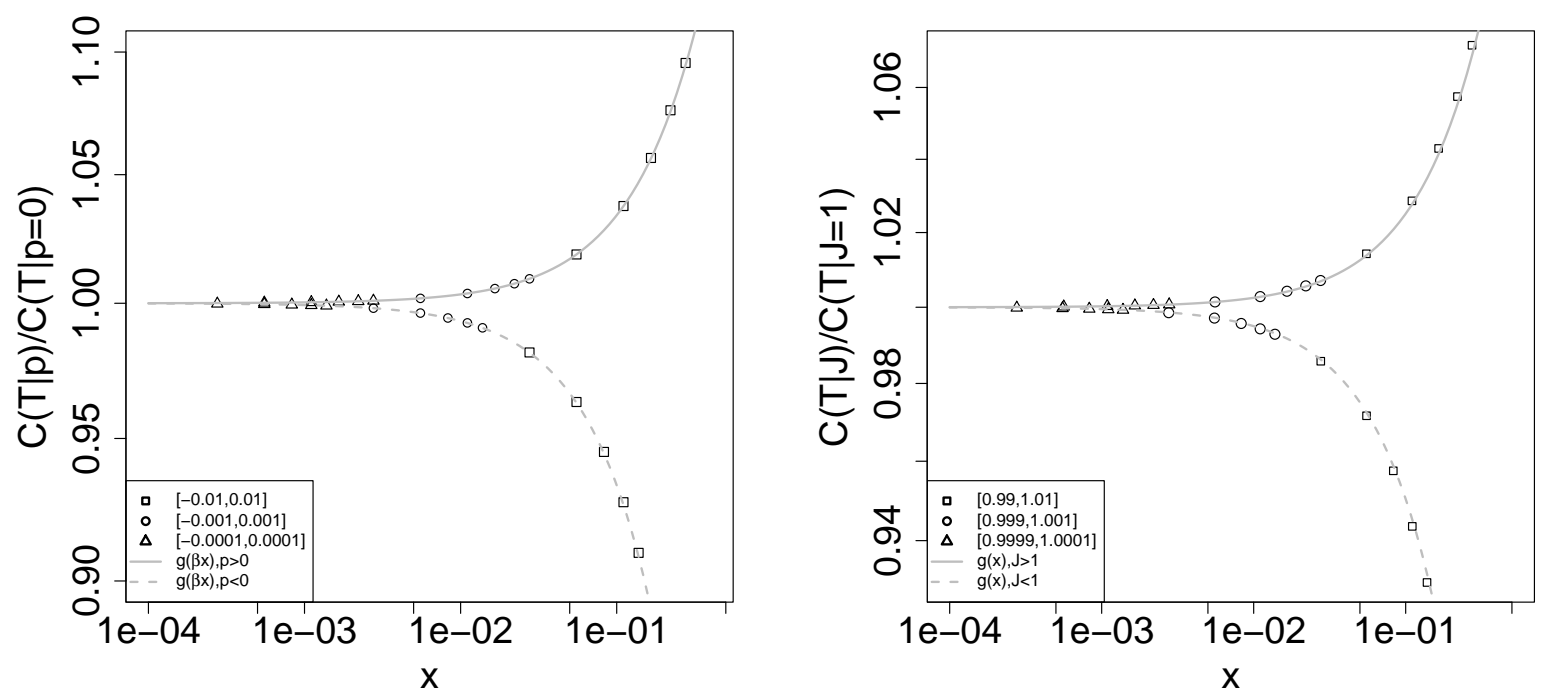

FIG. 3. (a) Plot of $C(T \mid p, 0) / C(T \mid 0,0)$ vs. $\quad x=T / \xi(p)$. We set $p \in[-0.01,0.01](\square)$, $[-0.001,0.001](\circ),[-0.0001,0.0001](\triangle), h=0$ and $T=10^{6} ; g(1.35 x)$ from Eq. (25) is plotted with gray solid $(p>0)$ and broken curves $(p<0)$. (b) Plot of $C(T \mid J, 0) / C(T \mid 1,0)$ vs. $x=T / \xi(J)$. We set $J \in[-0.99,1.01](\square),[-0.999,1.001](\circ),[0.9999,1.0001](\triangle), h=0$ and $T=10^{6} ; g(x)$ from Eq. (25) is plotted with gray solid $(J>1)$ and broken curves $(J<1)$.

be clearly seen that there are two phases: $c>0$ and $c=0$. When $p<0$, or $J<1$, $g(x)$ decays to zero for large $x$. As $C(T \mid p, 0) \simeq C(T \mid 0,0) g(x=\ln (T+1) / \xi(p, 0)$, we have $c=\lim _{T \rightarrow \infty} C(T \mid p, 0)=0$. When $p>0, g(x)$ grows as $x^{1 / 2}$ for large $x$. Then, as $C(T \mid 0,0) \simeq b \ln (T+1)^{-1 / 2}$, we have $c=\lim _{T \rightarrow \infty} C(T \mid p, 0)=b \ln (T+1)^{-1 / 2} g(\ln (T+$ 1) $/ \xi(p)) \propto \xi(p)^{-1 / 2}>0$.

We also check the universal function in the $h$ direction in Fig. 4. We adopt the same procedures as in the $p$ direction, and we estimate $C(T \mid 0, h) / C(T \mid 0,0)$ for $T=10^{6}$ and $h \in[0.0,0.01],[0.00,0.001]$. It can be seen that $g_{h}(x)$ in Eq. (26) does not describe $C(T \mid 0, h) / C(T \mid 0,0)$. The symmetry breaking field $h$ disrupts the universal asymptotic behavior of $C(T \mid h, 0)$. 


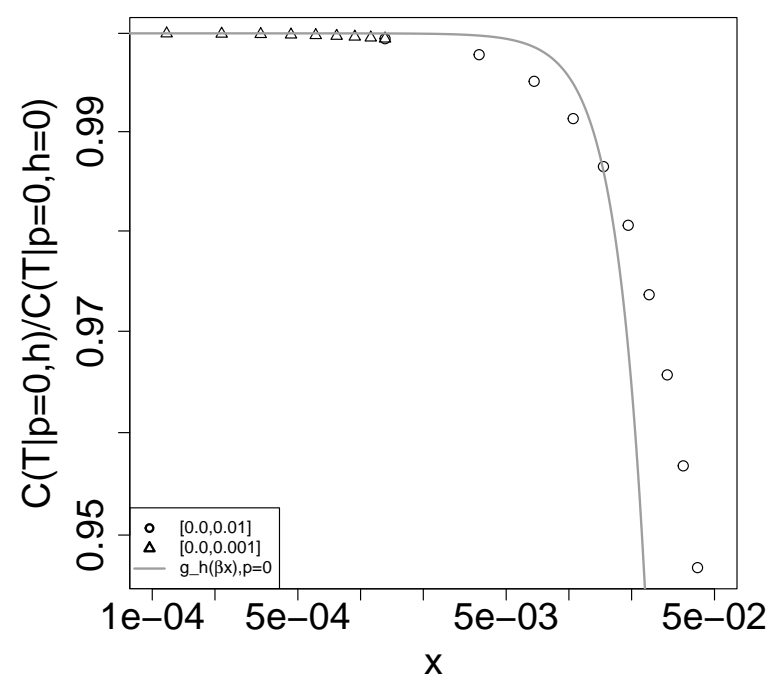

FIG. 4. Plot of $C(T \mid 0, h) / C(T \mid 0,0)$ vs. $x=T / \xi(h)$. We set $h \in[0.0,0.01](\circ),[0,0.001](\triangle), p=0$ and $T=10^{6} ; g_{h}\left(10^{2} x\right)$ from Eq. (26) is plotted with gray solid line.

\section{SUMMARY}

We studied the phase transition of a nonlinear Pólya urn using an SDE. We solved the initial value problem and analytically estimated the correlation function $C(T)$. By taking the scaling limit as $T \rightarrow \infty$ and $\xi \rightarrow \infty$, with $x=T / \xi$ fixed, we derived the universal function of the phase transition, which governs the asymptotic behavior of $C(T)$ near the critical point. We also numerically verified the results, where the phase transition of the system could be clearly observed.

Our study ascertained that scaling analysis is a powerful method to understand the nonequilibrium phase transition of non-linear Pólya urns. In addition, SDEs and the expansion about the strength of noise are also useful to understand the critical and universal behavior in non-equilibrium phase transition. A non-linear Pólya urn is one of the simplest systems in which time evolution is greatly affected by feedback. In nature, there are many stochastic

processes where some feedback mechanism plays crucial role, as Hawks process etc[19, 20]. We believe the scaling analysis continues to be promising approaches to understand the universal behavior of such systems. 


\section{ACKNOWLEDGMENTS}

The authors thanks Yugo Kagaya for useful discussions. This work was supported by JPSJ KAKENHI[Grant No. 17K00347].

[1] R. N. Mantegna and H. E. Stanley, Introduction to Econophysics: Correlations and Complexity in Finance (Cambridge University Press, Cambridge, 2007).

[2] W. B. Arthur, Econ. Jour. 99, 116 (1989).

[3] W. B. Arthur, Scientific American 262, 92 (1990).

[4] S. Bikhchandani, D. Hirshleifer, and I. Welch, J. Polit. Econ. 100, 992 (1992).

[5] J. Surowiecki, The Wisdom of Crowds (Doubleday, New York, 2004).

[6] B. Hill, D. Lane, and W. Sudderth, Ann. Probab. 8, 214 (1980).

[7] G. Pólya, Ann. Inst. Henri Poincaré 1, 117 (1931).

[8] R. Pemantle, Proc. Amer. Math. Soc. 113, 235 (1991).

[9] L. R. Anderson and C. A. Holt, Am. Econ. Rev. 87, 847 (1997).

[10] S. Mori, M. Hisakado, and T. Takahashi, Phys. Rev. E 86, 026109 (2012).

[11] M. Hino, Y. Irie, M. Hisakado, T. Takahashi, and S. Mori, J. Phys. Soc. Jpn. 85, 034002 (2016).

[12] S. Hod and U. Keshet, Phys. Rev. E 70, 015104 (2004).

[13] S. Mori and M. Hisakado, J. Phys. Soc. Jpn. 84, 054001 (2015).

[14] S. Mori and M. Hisakado, Phys. Rev. E 92, 052112 (2015).

[15] H. Hinrichsen, Adv. Phys. 49, 815 (2000).

[16] M. Hisakado and S. Mori, J. Phys. A 44, 275204 (2011).

[17] C. Gardiner, Stochastic Methods: A handbook for the Natural and Social Science, 4th ed. (Springer, Berlin, 2009).

[18] This is true at least up to $O\left(D^{2}\right)$ because the classical solutions $y_{0}^{ \pm}(t)$ respectively converge to $a_{ \pm}$.

[19] A. Hawkes, Journal of the Royal Statistical Society. Series B (Methodological) 33(3), 438 (1971).

[20] K. Kanazawa and D. Sornette, Phys. Rev. Resarch 2, 033442 (2020). 
[21] T. Huillet, J. Phys. A 41, 505005 (2008).

[22] M. Hisakado and S. Mori, J. Phys. A 45, 345002 (2012).

\section{Appendix A: Small Noise Approximation}

By substituting (9) into (8), a sequence of SDEs is obtained:

$$
\begin{aligned}
& d y_{0}=\frac{g\left(y_{0}, h\right)}{t+1} d t \\
& d y_{1}=\frac{g_{y}\left(y_{0}, h\right) y_{1}}{t+1} d t+\frac{1}{t+1} d W_{t}, \\
& d y_{2}=\frac{g_{y}\left(y_{0}, h\right) y_{2}+\frac{1}{2} g_{y y}\left(y_{0}, h\right) y_{1}^{2}}{t+1} d t .
\end{aligned}
$$

Equation (A1a) immediately yields Eq. (11). We now define $u_{k}^{ \pm}(t)=e^{-G^{ \pm}(t)} y_{k}^{ \pm}(t), k=1,2$,

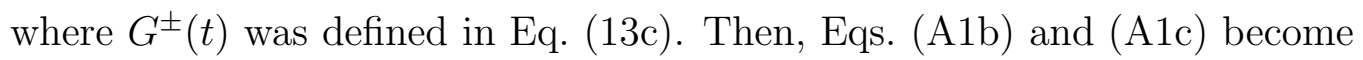

$$
\begin{aligned}
& d u_{1}^{ \pm}=\frac{e^{-G^{ \pm}(t)}}{t+1} d W_{t}, \\
& d u_{2}^{ \pm}=-3 \frac{e^{-G^{ \pm}(t)} y_{0}^{ \pm}(t) y_{1}^{ \pm}(t)^{2}}{t+1} d t .
\end{aligned}
$$

Thus, we have the closed-form solutions

$$
\begin{aligned}
& y_{1}^{ \pm}(t)=e^{G^{ \pm}(t)} \int_{0}^{t} \frac{e^{-G^{ \pm}(t)}}{t+1} d W_{t} \\
& y_{2}^{ \pm}(t)=-3 e^{G^{ \pm}(t)} \int_{0}^{t} \frac{e^{-G^{ \pm}(t)} y_{0}^{ \pm}(t) y_{1}^{ \pm}(t)^{2}}{t+1} d t .
\end{aligned}
$$

The expectation values are estimated as

$$
\begin{aligned}
E\left(y_{1}^{ \pm}(t)\right) & =0 \\
E\left(y_{2}^{ \pm}(t)\right) & =-3 e^{G^{ \pm}(t)} \int_{0}^{t} \frac{e^{-G^{ \pm}(t)} y_{0}^{ \pm}(t) E\left(y_{1}^{ \pm}(t)^{2}\right)}{t+1} d t \\
E\left(y_{1}^{ \pm}(t)^{2}\right) & =e^{2 G^{ \pm}(t)} \int_{0}^{t} \frac{e^{-2 G^{ \pm}(t)}}{(t+1)^{2}} d t
\end{aligned}
$$

Therefore, Eqs. (12a), (12b), (13a), and (13b) are obtained. 


\section{Appendix B: Zeros of the polynomial $g(y, h)$}

The zeros of the cubic polynomial $g(y, h)$ are given by the Cardano formula:

$$
a_{1}=\omega \alpha+\omega^{2} \beta, \quad a_{2}=\omega^{2} \alpha+\omega \beta, \quad a_{3}=\alpha+\beta,
$$

where $\omega=e^{2 \pi i / 3}$ is a primitive cube root of unity, and

$$
\alpha^{3}, \beta^{3}=\frac{h}{2} \pm \sqrt{\left(\frac{h}{2}\right)^{2}-\left(\frac{p}{3}\right)^{3}}, \quad \alpha \beta=\frac{p}{3} .
$$

The discriminant of the polynomial $g(y, h)$ is

$$
D=108\left\{\left(\frac{p}{3}\right)^{3}-\left(\frac{h}{2}\right)^{2}\right\}
$$

When $p \neq 0$, a convenient parametrization is

$$
\frac{h}{2}=\left(\frac{p}{3}\right)^{3 / 2} \cos \mu, \quad \therefore \alpha, \beta=\left(\frac{p}{3}\right)^{1 / 2} e^{ \pm i \mu / 3}
$$

Therefore, we have

$$
\begin{aligned}
& a_{1}=-2\left(\frac{p}{3}\right)^{1 / 2} \cos \frac{\pi-\mu}{3}, \\
& a_{2}=-2\left(\frac{p}{3}\right)^{1 / 2} \cos \frac{\pi+\mu}{3}, \\
& a_{3}=2\left(\frac{p}{3}\right)^{1 / 2} \cos \frac{\mu}{3} .
\end{aligned}
$$

We note that the parameter $\mu$ may be a complex number.

When the discriminant $D$ is positive, $g(y, h)$ has three distinct real zeros. Then, the order parameter $c(p, h)$ is given by

$$
c(p, h)=a_{3}-a_{1}=2 \sqrt{p} \cos \frac{\pi-2 \mu}{6}, \quad 0 \leq \mu<\frac{\pi}{2}
$$

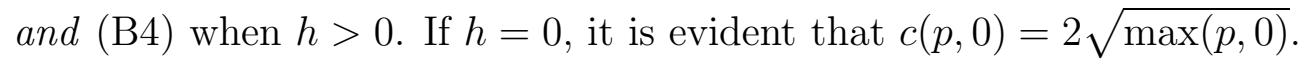




\section{Appendix C: Solutions of the SDE (86) in the Symmetric Domain}

Herein, we introduce a positive parameter $k$ into the function $g(y, h)$ :

$$
g(y, h)=-k y^{3}+k p y+k h
$$

If $h=0$, after some arithmetic, we can show that

$$
\begin{aligned}
y_{0}^{ \pm}(t)= & \pm \sqrt{\frac{p}{1-\beta(t+1)^{-2 k p}}}, \quad \beta=1-4 p \\
E\left(y_{1}^{ \pm}(t)^{2}\right)= & \frac{1}{\left\{1-\beta(t+1)^{-2 k p}\right\}^{3}}\left\{\frac{(t+1)^{-1}}{4 k p-1}-\frac{3 \beta(t+1)^{-1-2 k p}}{2 k p-1}-3 \beta^{2}(t+1)^{-1-4 k p}\right. \\
& \left.+\frac{\beta^{3}(t+1)^{-1-6 k p}}{2 k p+1}-\left(\frac{1}{4 k p-1}-\frac{3 \beta}{2 k p-1}-3 \beta^{2}+\frac{\beta^{3}}{2 k p+1}\right)(t+1)^{-4 k p}\right\},
\end{aligned}
$$

for $p \neq 0$, and

$$
\begin{aligned}
y_{0}^{ \pm}(t)= & \frac{1}{2 \sqrt{\tau(t)}}, \quad \tau(t)=\frac{k}{2} \ln (t+1)+1 \\
E\left(y_{1}^{ \pm}(t)^{2}\right)= & \left\{\left(1+\frac{3 k}{2}+\frac{3 k^{2}}{2}+\frac{3 k^{3}}{4}\right) \tau(t)^{-3}\right. \\
& \left.-\left(1+\frac{3 k}{2 \tau(t)}+\frac{3 k^{2}}{2 \tau(t)^{2}}-\frac{3 k^{3}}{4 \tau(t)^{3}}\right)(t+1)^{-1}\right\},
\end{aligned}
$$

for $p=0$. We note that the asymptotic behavior of the variance is estimated as follows:

$$
V\left(y^{ \pm}(t)\right) \sim \begin{cases}\frac{(t+1)^{-1}}{4 k p-1}, & k p>\frac{1}{4}, \\ (t+1)^{-1} \ln (t+1), & k p=\frac{1}{4}, \\ \left(\frac{1}{1-4 k p}-\frac{3 \beta}{1-2 k p}+3 \beta^{2}-\frac{\beta^{3}}{2 k p+1}\right)(t+1)^{-4 k p}, & 0<k p<\frac{1}{4}, \\ \left(1+\frac{3 k}{2}+\frac{3 k^{2}}{2}+\frac{3 k^{3}}{4}\right)\{\ln (t+1)\}^{-3}, & k p=0, \\ \left(\frac{\beta^{-3}}{4 k p-1}-\frac{3 \beta^{-2}}{2 k p-1}-3 \beta^{-1}+\frac{1}{2 k p+1}\right)(t+1)^{-2 k|p|}, & -\frac{1}{2}<k p<0, \\ (t+1)^{-1} \ln (t+1), & k p=-\frac{1}{2}, \\ \frac{(t+1)^{-1}}{-2 k p-1}, & k p<-\frac{1}{2} .\end{cases}
$$

In [12, 21, 22], phase transition in a binary sequence system was discussed. This system 
is a nonlinear Pólya urn with (in our notation) the probability function

$$
f(z, 0)=\frac{1}{2}\left(1-\lambda \frac{1-2 z}{1+t_{0} / t}\right), \quad-1<\lambda<1,
$$

where $t_{0}$ is initial time. When $t$ is large, we have

$$
\begin{aligned}
& f(z, 0) \simeq \frac{1}{2}+\lambda\left(z-\frac{1}{2}\right), \\
& \therefore f(z, 0)-z \simeq(\lambda-1) y,
\end{aligned}
$$

where $y=z-1 / 2$ has been defined. Thus, by keeping $k p=\lambda-1$ constant and taking the limit as $k \rightarrow+0$, the variance converges to

$$
V\left(y^{ \pm}(t)\right) \sim \begin{cases}\frac{1}{2 \lambda-1}(t+1)^{2(\lambda-1)}, & -\frac{1}{2}<\lambda-1<0, \\ (t+1)^{-1} \ln (t+1), & \lambda-1=-\frac{1}{2}, \\ \frac{1}{1-2 \lambda}(t+1)^{-1}, & \lambda-1<-\frac{1}{2} .\end{cases}
$$

As $z$ is the proportion of $+($ or -$)$ symbols, we introduce a scaled random variable $w=t z$ that represents the number of $+($ or -$)$ symbols. Its variance is

$$
V\left(w^{ \pm}(t)\right) \sim \begin{cases}\frac{1}{2 \lambda-1}(t+1)^{2 \lambda}, & -\frac{1}{2}<\lambda-1<0, \\ (t+1) \ln (t+1), & \lambda-1=-\frac{1}{2}, \\ \frac{1}{1-2 \lambda}(t+1), & \lambda-1<-\frac{1}{2} .\end{cases}
$$

Thus, we conclude that $\lambda=\frac{1}{2}$ is the critical point, and that $\frac{1}{2}<\lambda<1$ is a super-diffusion phase. 